\title{
YAP knockdown inhibits proliferation and induces apoptosis of human prostate cancer DU145 cells
}

\author{
XIN JIN* , WENCHAO ZHAO* ${ }^{*}$ PENG ZHOU* and TIANLI NIU \\ Department of Urology, The Affiliated Taizhou People's Hospital of Nantong University, Taizhou, Jiangsu 225300, P.R. China
}

Received September 27, 2016; Accepted August 16, 2017

DOI: $10.3892 / \mathrm{mmr} .2017 .8352$

\begin{abstract}
The role of yes-associated protein (YAP) in human prostate cancer DU145 cells and its underlying molecular mechanisms were explored in the present study. Initially, the expression levels of YAP were detected in DU145 cells, which revealed that YAP was highly expressed in these cells. To investigate the role of YAP in DU145 cells, a stable YAP-silenced DU145 cell line was generated using YAP-small interfering RNA. Reverse transcription-quantitative polymerase chain reaction and western blotting were performed for mRNA and protein detection, respectively. An MTT assay and flow cytometry were performed to investigate the proliferation and apoptosis of DU145 cells. The results demonstrated that YAP knockdown significantly decreased the proliferative ability of DU145 cells, whereas the percentage of apoptotic cells was markedly increased, compared with the control. In addition, the mRNA and protein expression levels of connective tissue growth factor and cysteine-rich angiogenic factor 61 were notably decreased, the ratio of B-cell lymphoma 2 (Bcl-2)/Bcl-2-associated X protein (Bax) was significantly reduced, and the expression levels of caspase 3 were significantly decreased within YAP-silenced DU145 cells. In conclusion, YAP knockdown reduced the proliferation and induced apoptosis of DU145 cells. Therefore, the gene transcription and protein expression of YAP may be involved in the development of prostate cancer and may be considered a potential target for the treatment of such cancers.
\end{abstract}

\section{Introduction}

Prostate cancer ( $\mathrm{PCa}$ ) is the most common malignancy that is frequently diagnosed in males, and is a major cause of

Correspondence to: Dr Tianli Niu, Department of Urology, The Affiliated Taizhou People's Hospital of Nantong University, 210 Yingchun Road, Taizhou, Jiangsu 225300, P.R. China E-mail: niut10905@163.com

\section{${ }^{*}$ Contributed equally}

Key words: yes-associated protein, Hippo pathway, prostate cancer, proliferation, apoptosis cancer-associated mortality worldwide (1). Androgen deprivation therapy is the most effective treatment for advanced $\mathrm{PCa}$; however, most patients with PCa develop castration-resistant prostate cancer (CRPC) due to therapy-associated resistance $(2,3)$. Unfortunately, CRPC remains incurable (2) and the underlying mechanisms of CRPC development are yet to be revealed.

The Hippo signaling pathway, which was originally identified in fruit flies, is involved in tumor development via the regulation of cell proliferation and apoptosis (3-7). The core components of the Hippo signaling pathway within mammals comprise protein kinases, including mammalian sterile 20-like 1 and 2, large tumor suppressor 1 and 2, and the adaptor proteins WW domain-containing protein and Mps one binder 1 . The core functions of the Hippo signaling pathway serve tumor-suppressing roles via yes-associated protein (YAP), and TAZ phosphorylation and inactivation. YAP, which is a $65-\mathrm{kDa}$ protein, is an effector protein of the Hippo signaling pathway and is a transcriptional co-activator of numerous transcription factors. Previous studies have confirmed that YAP functions as an oncogenic protein in mammalian cells via promoting cell growth (8-10). Additionally, YAP overexpression has been associated with various human cancers, including PCa, and breast, ovarian, lung, liver and gastric cancer (11-17).

Increasing evidence has suggested the important roles of YAP in regulating PCa cell behavior $(18,19)$. To the best our knowledge, no previous study has focused on the role of YAP within human PCa DU145 cells. Therefore, the role of YAP in human PCa DU145 cells and the underlying molecular mechanisms were explored in the present study.

\section{Materials and methods}

Materials. The human PCa DU145 cells and RWPE-1 cells were obtained from the American Type Culture Collection (Manassas, VA, USA); serum-free keratinocyte medium (K-SFM) was obtained from Gibco (Thermo Fisher Scientific, Inc., Waltham, MA, USA); the RPMI-1640 medium, fetal bovine serum (FBS) and polyvinylidene fluoride (PVDF) membrane were obtained from Sigma-Aldrich (Merck KGaA, Darmstadt, Germany). Primary antibodies against connective tissue growth factor (CTGF, cat. no. sc-14939 were purchased from Santa Cruz Biotechnology, Inc., Dallas, TX, USA, while, cysteine-rich angiogenic inducer 61 antibodies (CYR61, cat. no. 14476, 1:1,000), B-cell lymphoma 2 antibodies 
(Bcl-2, cat. no. 2872, 1:1,000), Bcl-2-associated X protein antibodies (Bax, cat. no. 2774, 1:1,000), caspase 3 antibodies (cat. no. 9662, 1:1,000), GAPDH antibodies (cat. no. 5174, 1:1,000) and secondary antibodies (cat. no. 7076, 1:1,000) were obtained from Cell Signaling Technology Inc., (Danvers, MA, USA); MTT and enhanced chemiluminescence (ECL) Plus reagent were obtained from Eli Lilly \& Co., (Indianapolis, IN, USA); Annexin V-fluorescein isothiocyanate (FITC) apoptosis detection kit was obtained from Vazyme Biotech Co. (Nanjing, China). YAP-small interfering RNA (YAP-siRNA, cat. no. sc-38637), control (Con)-siRNA (cat. no. sc-37007) and the siRNA Transfection Reagent (cat. no. sc-29528) were obtained from Santa Cruz Biotechnology, Inc.

Cell culture. Human PCa DU145 cells were cultured in RPMI-1640 supplemented with 10\% FBS and 1\% penicillin-streptomycin. RWPE-1 cells were cultured in $10 \mathrm{ml}$ complete SFM containing $5 \mathrm{ng} / \mathrm{ml}$ recombinant epidermal growth factor (CYT-217, ProSpec-Tany TechnoGene Ltd., East Brunswick, NJ, USA), $50 \mu \mathrm{g} / \mathrm{ml}$ bovine pituitary extract (CC023; macgene.bioon.com.cn/), $100 \mathrm{IU} / \mathrm{ml}$ penicillin and $100 \mathrm{IU} / \mathrm{ml}$ streptomycin. Cells were incubated in standard cell culture conditions $\left(5 \% \mathrm{CO}_{2}, 95 \%\right.$ humidity) at $37^{\circ} \mathrm{C}$. Human PCa DU145 cells were passaged every 2-3 days, and RWPE-1 cells were passaged every $\sim 5$ days.

Cell transfection. Human PCa DU145 cells were plated in a 6 -well plate 1 day prior to transfection, the transfection assay was performed once the cells reached $60-70 \%$ confluence. DU145 cells $\left(2 \times 10^{5}\right.$ cells/well) were transfected with YAP-siRNA or Con-siRNA $(1 \mu \mathrm{g})$ using $30 \mu \mathrm{l}$ transfection reagent plus and incubated at $37^{\circ} \mathrm{C}$ for $6 \mathrm{~h}$ in $5 \% \mathrm{CO}_{2}$ incubator, according to the manufacturer's protocol. A total of $24 \mathrm{~h}$ following transfection, the transfected DU145 cells were used for subsequent experiments, and were collected for protein analysis after $72 \mathrm{~h}$ incubation.

RNA isolation and reverse transcription-quantitative polymerase chain reaction $(R T-q P C R)$. Total RNA was extracted from human PCa DU145 and RWPE-1 cells using TRIzol reagent (Invitrogen; Thermo Fisher Scientific, Inc.) according to the manufacturer's protocol. To determine the mRNA expression levels within the cells, RT was performed using PrimeScript ${ }^{\mathrm{TM}}$ RT reagent kit (Takara Bio Inc., Otsu, Japan) according to the manufacture's protocol. For qPCR, SYBR $^{\circledR}$ Premix Ex Taq ${ }^{\mathrm{TM}}$ II (Takara Bio Inc.) was used and all reactions were performed in triplicate with the following conditions: $95^{\circ} \mathrm{C}$ for $10 \mathrm{~min}$, followed by 40 cycles at $95^{\circ} \mathrm{C}$ for $15 \mathrm{sec}$ and $72^{\circ} \mathrm{C}$ for $30 \mathrm{sec}, 78^{\circ} \mathrm{C}$ for $1.5 \mathrm{~min}$ for $35 \mathrm{cycles}$, after which samples were stored at $4^{\circ} \mathrm{C}$. GAPDH was used as an internal control. The $2^{-\Delta \Delta \mathrm{Cq}}$ method was performed to analyze the relative amounts of each transcript (20). The qPCR primers are presented in Table I.

Western blot analysis. Total cellular protein from human PCa DU145 and RWPE-1 cells was extracted using radioimmunoprecipitation assay buffer (P0013B, Beyotime Biotechnology, Nanjing, China) and SDS-PAGE analysis was performed to separate the total protein. Protein concentration was determined by bicinchoninic protein assay kit. Proteins $(25 \mu \mathrm{g})$ were resolved by $10 \%$ sodium dodecyl sulfate polyacrylamide gels and were then transferred to a polyvinylidene membrane. The membrane was blocked with $5 \%$ skim milked for $1 \mathrm{~h}$ at room temperature. Subsequently, the membrane was blotted overnight at $4^{\circ} \mathrm{C}$ with the following primary antibodies: CTGF (1:1,000 dilution), CYR61 (1:1,000 dilution), Bcl-2 (1:1,000 dilution), Bax (1:1,000 dilution), caspase 3 (1:1,000 dilution) and GAPDH (1:2,000 dilution). The membrane was then incubated with horseradish peroxidase-conjugated anti-mouse and anti-rabbit immunoglobulin $\mathrm{G}$ secondary antibodies (1:5,000 dilution) at room temperature for $2 \mathrm{~h}$. Protein bands were observed using enhanced chemiluminescence Plus reagent prior to imaging and analysis. MTT assay. Cell proliferation was detected using an MTT assay. YAP-siRNA and Con-siRNA were transfected into DU145 cells. Subsequently, log-phase human PCa DU145 cells were harvested with $0.25 \%$ trypsin and seeded in 96 -well plates $\left(5 \times 10^{3}\right.$ cells/well). After $24 \mathrm{~h}$ incubation at $37^{\circ} \mathrm{C}$ with $5 \% \mathrm{CO}_{2}$, MTT was added to the cell culture medium and incubated for $4 \mathrm{~h}$ at $37^{\circ} \mathrm{C}$. Then DMSO was employed for formazan crystals dissolving. Optical density was detected at $490 \mathrm{~nm}$ using a spectrophotometer. Each experiment was repeated in triplicate and data were displayed as the mean \pm standard deviation.

Apoptosis analysis. To detect alterations in cell apoptosis, an Annexin V-FITC apoptosis detection kit was utilized. DU145 cells were transfected with YAP-siRNA or Con-siRNA for 24 h. Following transfection, cells were rinsed with cold PBS. Cells (5x10 5 cells/well) were then labeled with Annexin V-FITC and propidium iodide, according to the manufacturer's protocol. Flow cytometry (BD Biosciences, Franklin Lakes. NJ, USA) was used for cell apoptosis analysis. Version 2.5 WinMDI (Purdue University Cytometry Laboratories; www. cyto.purdue.edu/flowcyt/software/Catalog.htm) was applied for data analysis. Each test was repeated in triplicate.

Statistical analysis. All tests were performed in triplicate. Data are displayed as the mean \pm standard deviation. SPSS 17.0 statistical software (SPSS, Inc., Chicago, IL, USA) was applied for all statistical analyses. A Student's t-test was used to analyze the difference between the two groups. $\mathrm{P}<0.05$ was considered to indicate a statistically significant difference.

\section{Results}

YAP expression in human PCa DU145 cells. Firstly, the mRNA and protein expression levels of YAP were detected in human PCa DU145 cells via RT-qPCR and western blotting, respectively. As demonstrated in Fig. 1, the mRNA and protein expression levels of YAP were significantly higher in human PCa DU145 cells compared with in normal prostate epithelial RWPE-1 cells. Based on these results, the expression levels of YAP were significantly increased in human PCa DU145 cells.

mRNA and protein expression levels of YAP in DU145 cells following cell transfection. To investigate the role of YAP in human PCa DU145 cells, a stable YAP-silenced DU145 cell line was generated using YAP-siRNA. The results of RT-qPCR, compared with the blank group, indicated that the expression levels of YAP within YAP-siRNA-transfected cells 
Table I. Primer sequences for polymerase chain reaction.

\begin{tabular}{ll}
\hline Gene & \multicolumn{1}{c}{ Primer sequence (5'-3') } \\
\hline YAP-Forward & ACGTTCATCTGGGACAGCAT \\
YAP-Reverse & GTTGGGAGATGGCAAAGACA \\
CTGF-Forward & TTGGCAGGCTGATTTCTAGG \\
CTGF-Reverse & GGTGCAAACATGTAACTTTTGG \\
Cyr61-Forward & CCCGTTTTGGTAGATTCTGG \\
Cyr61-Reverse & GCTGGAATGCAACTTCGG \\
Bcl-2-Forward & ATGTGTGTGGAGAGCGTCAA \\
Bcl-2-Reverse & ACAGTTCCACAAAGGCATCC \\
Bax-Forward & GGCCCACCAGCTCTGAGCAGA \\
Bax-Reverse & GCCACGTGGGCGTCCCAAAGT \\
Caspase 3-Forward & CTGGTTGGCGTCGCCTTG \\
Caspase 3-Reverse & GAATCCACTGAGTTTTCAG \\
GAPDH-Forward & CTTTGGTATCGTGGAAGGACTC \\
GAPDH-Reverse & GTAGAGGCAGGGATGATGTTCT
\end{tabular}

Bax, B-cell lymphoma 2-associated X protein; Bcl-2, B-cell lymphoma 2; CTGF, connective tissue growth factor; CYR61, cysteine-rich angiogenic inducer 61 ; YAP, yes-associated protein.

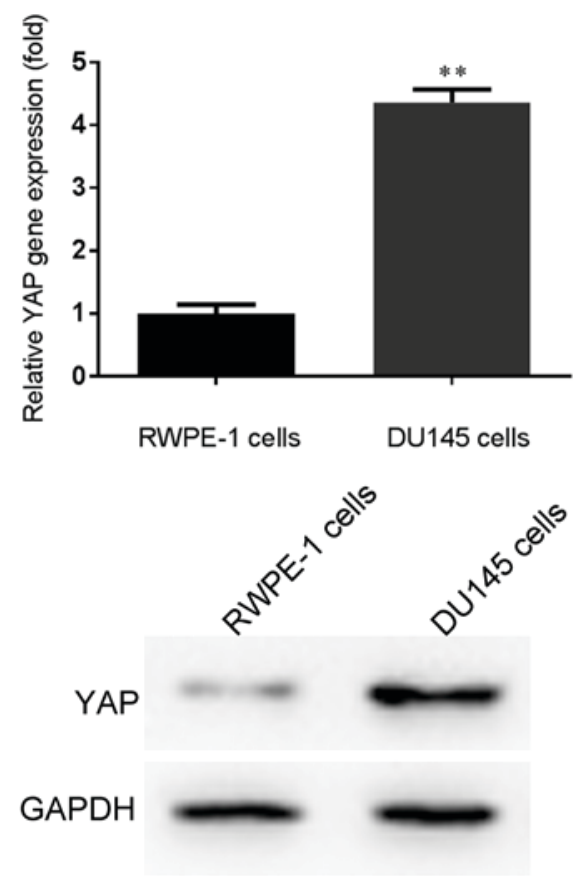

Figure 1. Expression levels of YAP in DU145 and RWPE-1 cells. mRNA and protein expression levels of YAP were determined in DU145 and RWPE-1 cells by reverse transcription-quantitative polymerase chain reaction and western blot analysis, respectively. All data are presented as the mean \pm standard deviation of three independent experiments. ${ }^{* *} \mathrm{P}<0.01$. YAP, yes-associated protein.

were markedly decreased, whereas Con-siRNA did not affect YAP expression. Reduced YAP protein expression was also revealed by western blotting in the YAP-siRNA group (Fig. 2). These data indicated that YAP-siRNA may effectively inhibit YAP expression.
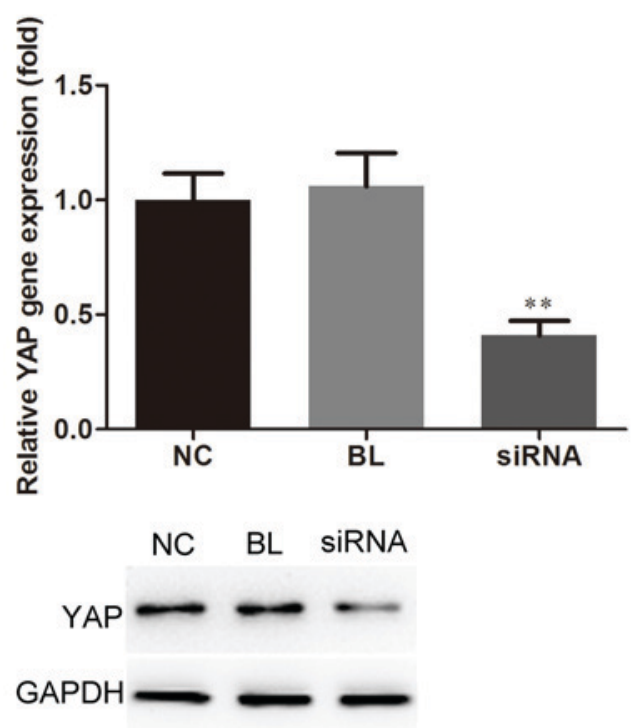

Figure 2. YAP was effectively inhibited by YAP-siRNA. DU145 cells were transfected with YAP-siRNA or Con-siRNA. A total of $24 \mathrm{~h}$ post-transfection, the mRNA and protein expression levels of YAP in DU145 cells were determined by reverse transcription-quantitative polymerase chain reaction and western blot analysis, respectively. NC, cells transfected with Con-siRNA; BL, cells without treatment; siRNA, cells transfected with YAP-siRNA. All data are presented as the mean \pm standard deviation of three independent experiments. ${ }^{* *} \mathrm{P}<0.01$ vs. the negative control. BL, blank; NC, negative control; siRNA, small interfering RNA; YAP, yes-associated protein.

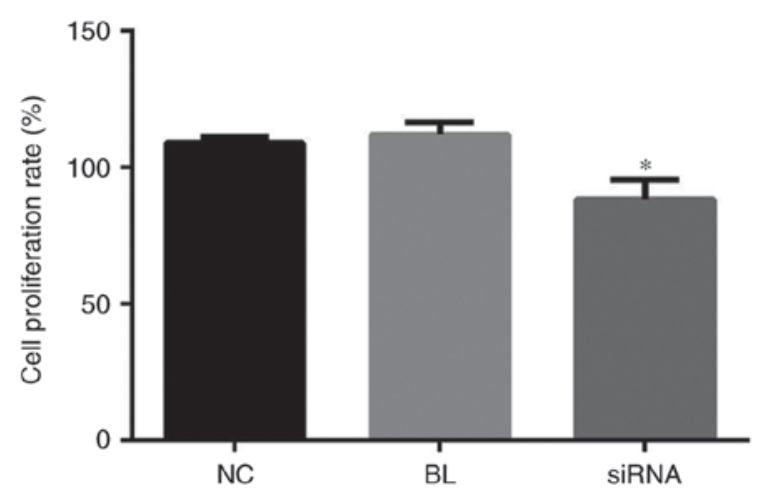

Figure 3. YAP inhibition inhibits the proliferation of DU145 cells. Cell proliferation was analyzed using an MTT assay $24 \mathrm{~h}$ after DU145 cells were transfected with YAP-siRNA or Con-siRNA. NC, cells transfected with Con-siRNA; BL, cells without treatment; siRNA, cells transfected with YAP-siRNA. ${ }^{*} \mathrm{P}<0.05$. All data are presented as the mean \pm standard deviation of three independent experiments. BL, blank; NC, negative control; siRNA, small interfering RNA; YAP, yes-associated protein.

Downregulation of YAP reduces the proliferative ability of DU145 cells. To investigate the effects of YAP expression on DU145 cell proliferation, YAP-siRNA and Con-siRNA were transfected into DU145 cells, and an MTT assay was performed. The results of the present study demonstrated that compared with the blank and control group, cell proliferation was markedly inhibited in YAP-siRNA-transfected DU145 cells. Furthermore, colony formation ability of cells was measured by MTT assay. The results indicated that YAP knockdown significantly suppressed the DU145 cell colony-forming ability (Fig. 3). These results indicated 

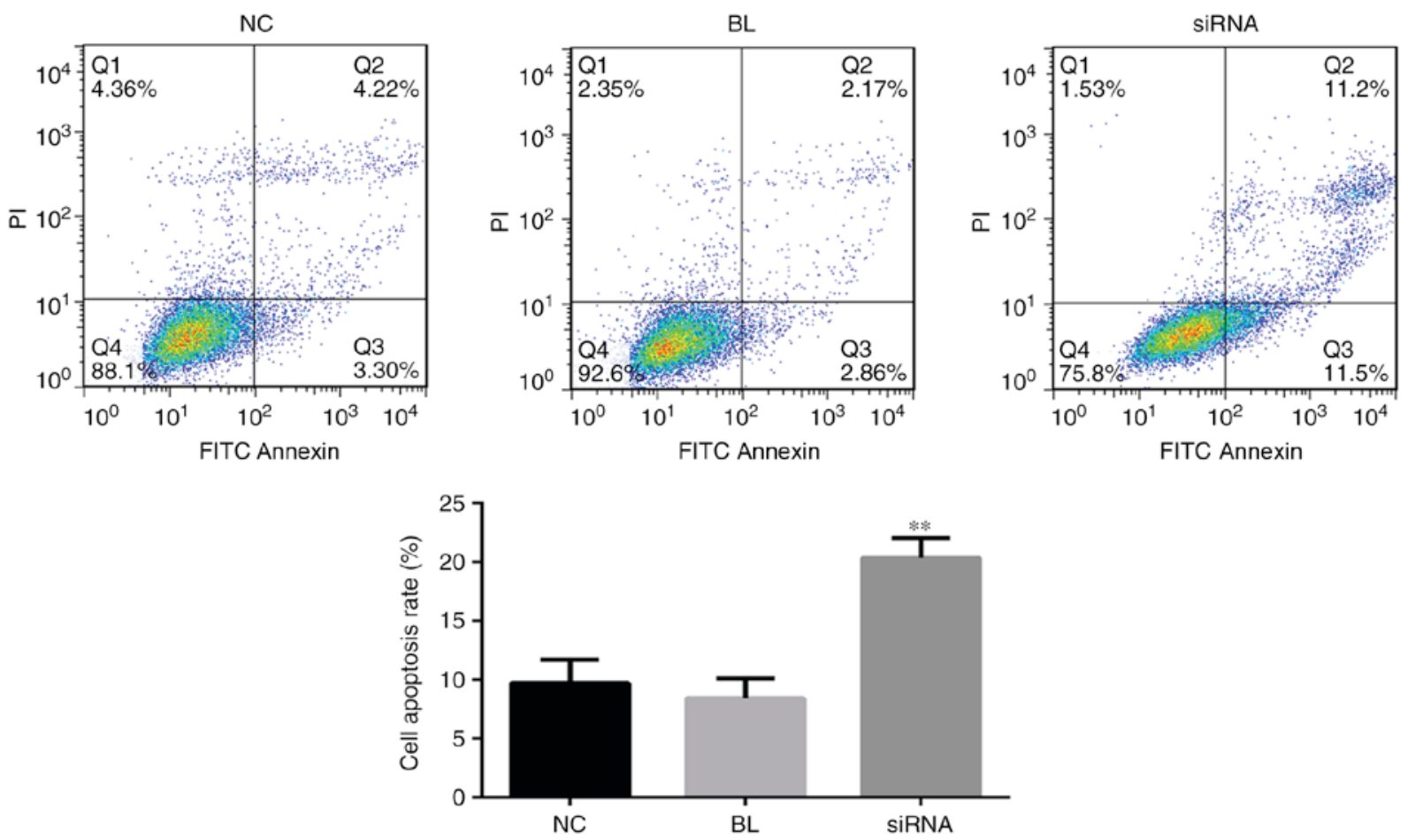

Figure 4. YAP inhibition induces the apoptosis of DU145 cells. A total of $24 \mathrm{~h}$ after DU145 cells were transfected with YAP-siRNA or Con-siRNA, flow cytometry was performed to detect cell apoptosis measuring the sum of cell percentage in quadrants Q2 and Q3. NC, cells transfected with Con-siRNA; BL, cells without any treatment; siRNA, cells transfected with YAP-siRNA. All data are presented as the mean \pm standard deviation of three independent experiments. ${ }^{* *} \mathrm{P}<0.01$. BL, blank; FITC, fluorescein isothiocyanate; NC, negative control; PI, propidium iodide; siRNA, small interfering RNA; YAP, yes-associated protein.

that YAP downregulation reduced the proliferation and colony-forming ability of DU145 cells.

Downregulation of YAP increases apoptosis of DU145 cells. The observed growth inhibition in YAP-siRNA-transfected DU145 cells was investigated using an Annexin V-FITC apoptosis detection kit for cell apoptosis via flow cytometry. The results revealed that YAP knockdown significantly induced DU145 cell apoptosis compared with in the control group (Fig. 4).

Downregulation of YAP alters the expression of CTGF, CYR61, Bcl-2, Bax and caspase 3. CTGF and CYR61 are downstream genes of YAP in the Hippo signaling pathway, and are regulated by YAP proteins. It has been reported that CTGF and CYR61 serve important roles in promoting cell proliferation, migration and invasion in cancer (21). Following transfection of DU145 cells with YAP-siRNA, the mRNA and protein expression levels of CTGF and CYR61 genes were significantly reduced compared with in Con-siRNA-transfected cells (Fig. 5).

Western blotting and RT-qPCR were performed to further explore the mechanism of YAP knockdown-induced cell apoptosis through the expression levels of the apoptosis-associated proteins Bax, Bcl-2 and caspase 3. The results of the present study suggested that the Bcl-2/Bax ratio was markedly lower in DU145 cells transfected with YAP-siRNA compared with in cells transfected with Con-siRNA. As expected, the expression levels of caspase 3 were notably decreased following YAP-siRNA transfection (Fig. 5).

\section{Discussion}

The major findings of the present study included the high expression of YAP in human PCa DU145 cells compared with in normal prostate epithelial RWPE-1 cells. Silencing of the YAP gene in DU145 cells was associated with reduced proliferative ability and a significant increase in apoptosis. The expression levels of tumor-associated genes, including CTGF, CYR61, Bcl-2, BAX and caspase 3, were also altered in DU145 cells when YAP expression was knocked down. These findings suggested that YAP may function as an oncogene in human PCa.

An imbalance between cell proliferation and apoptosis may result in cancer (22). The Hippo signaling pathway serves important roles in the regulation of tumor cell and tissue growth. YAP, which is a core component of the Hippo-YAP signaling pathway, has been confirmed to be associated with tumor metastasis, grade and stage $(23,24)$. It has also been reported that the function of YAP may be dysregulated in numerous cancers, serving oncogenic and tumor suppressive roles; previous studies have also indicated that YAP depletion may inhibit the growth and metastasis of tumor cells (25-30).

In the present study, YAP expression was detected in human PCa DU145 cells, and the effects of YAP knockdown on the proliferative ability and apoptosis of DU145 cells were determined in vitro. The results suggested that YAP was highly expressed in DU145 cells. However, the proliferative ability of cells was reduced and cell apoptosis was increased following YAP downregulation. The underlying mechanism of the regulation of cell behavior via YAP knockdown was 
A

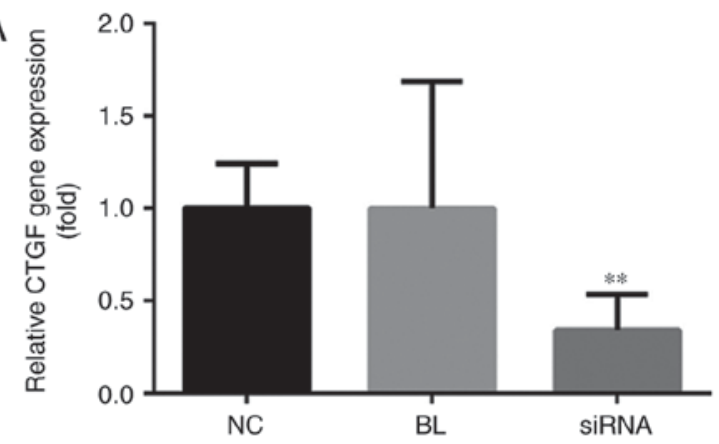

C

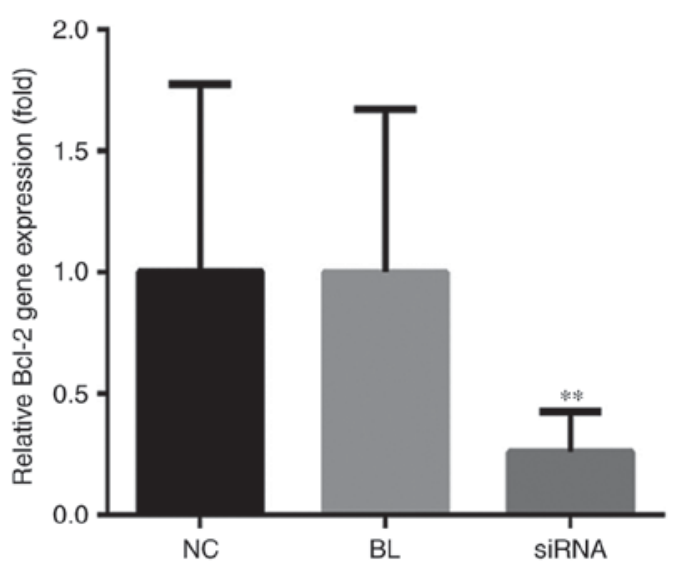

$\mathrm{E}$

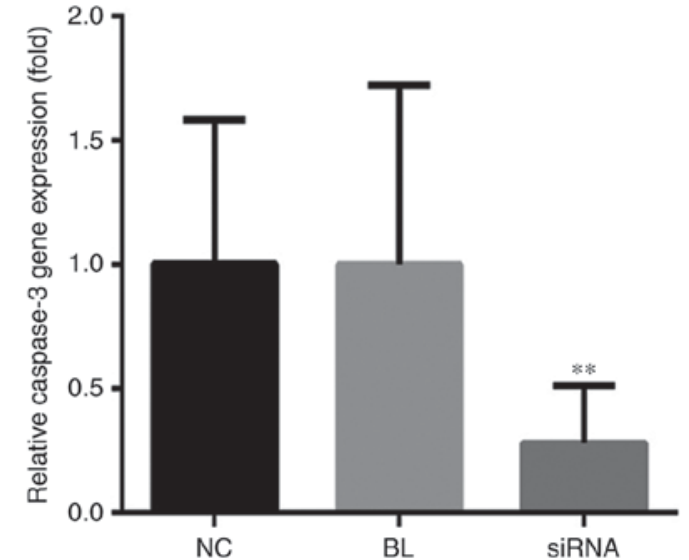

B

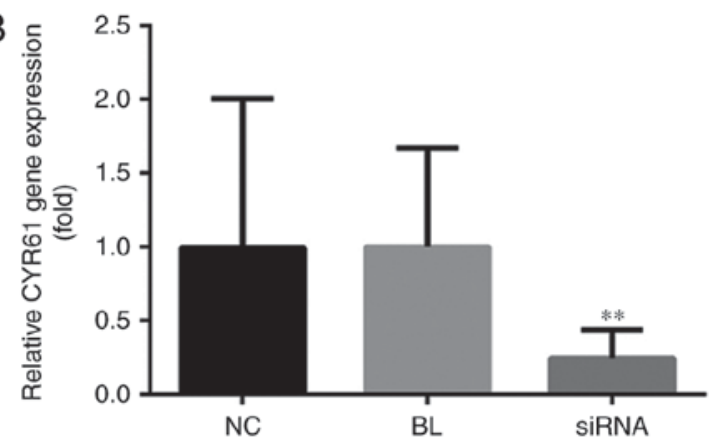

D
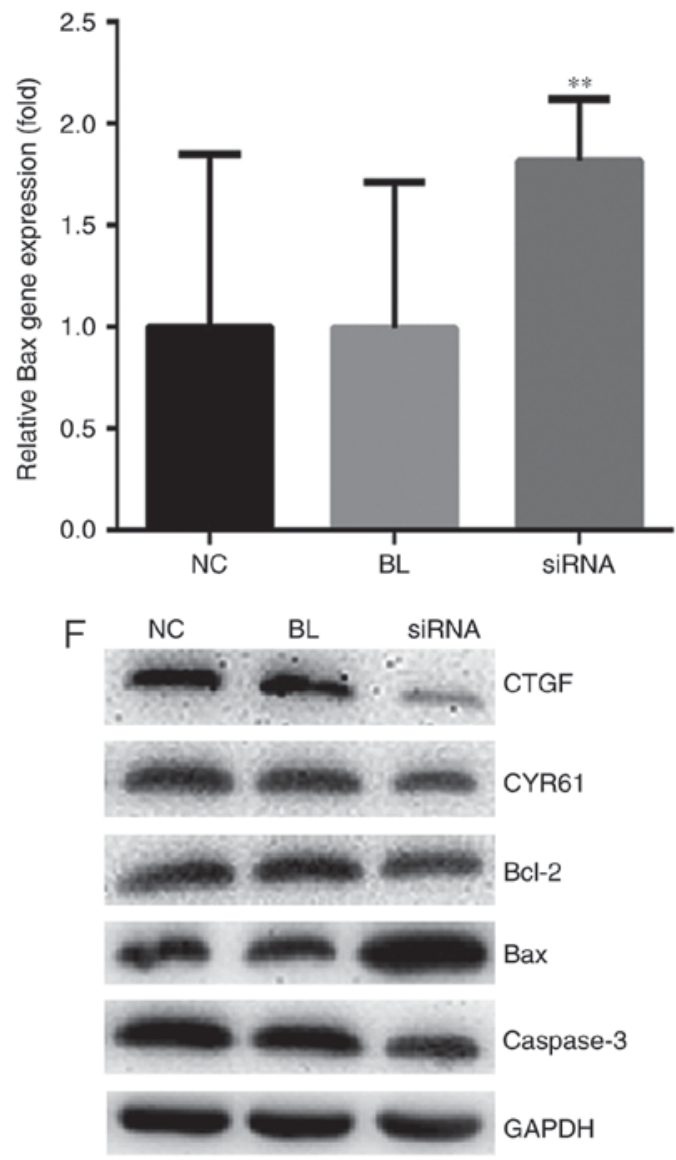

Figure 5. YAP inhibition alters the expression of related genes in DU145 cells. DU145 cells were transfected with YAP-siRNA or Con-siRNA. A total of $24 \mathrm{~h}$ after transfection, CTGF, CYR61, Bcl-2, Bax and caspase 3 expression levels were measured using (A-E) reverse transcription-quantitative polymerase chain reaction and $(\mathrm{F})$ western blotting, respectively. NC, cells transfected with Con-siRNA; BL, cells without any treatment; siRNA, cells transfected with YAP-siRNA. ${ }^{* *} \mathrm{P}<0.01$. Bax, B-cell lymphoma 2-associated X protein; Bcl-2, B-cell lymphoma 2; CTGF, connective tissue growth factor; CYR61, cysteine-rich angiogenic inducer 61; siRNA, small interfering RNA; YAP, yes-associated protein.

investigated through the detection of tumor-associated genes. CYR61 and CTGF are matricellular proteins involved in numerous physiological and pathological processes, including carcinogenesis, which serve critical roles in regulating tumor cell growth (31). Following YAP knockdown in DU145 cells, decreased expression levels of CTGF and CYR61 were detected. Furthermore, the expression levels of apoptosis-associated proteins (Bax, Bcl-2 and caspase 3) were analyzed. The data revealed that the $\mathrm{Bcl}-2 / \mathrm{Bax}$ ratio was markedly reduced in YAP-silenced DU145 cells and the expression levels of caspase 3 were significantly decreased compared with in the control group.
In conclusion, these results indicated that YAP knockdown suppressed the proliferation, and induced apoptosis of human PCa DU145 cells. Therefore, the YAP gene may be associated with tumorigenesis and the development of PCa which may serve as a potential future treatment target.

\section{Acknowledgements}

The authors would like to thank Professor Ming Chen and Professor Shuqiu Chen (Zhongda Hospital Affiliated to Southeast University, Nanjing, Jiangsu, China), and their team, for help with the experiments and data collection. 


\section{References}

1. Foley $\mathrm{C}$ and Mitsiades $\mathrm{N}$ : Moving beyond the androgen receptor (AR): Targeting AR-interacting proteins to treat prostate cancer. Horm Cancer 7: 84-103, 2016.

2. Chen Y, Sawyers CL and Scher HI: Targeting the androgen receptor pathway in prostate cancer. Curr Opin Pharmacol 8: 440-448, 2008.

3. Zhao B, Li L, Lei Q and Guan KL: The Hippo-YAP pathway in organ size control and tumorigenesis: An updated version. Genes Dev 24: 862-874, 2010.

4. Pan D: The hippo signaling pathway in development and cancer. Dev Cell 19: 491-505, 2010.

5. Park HW and Guan KL: Regulation of the Hippo pathway and implications for anticancer drug development. Trends Pharmacol Sci 34: 581-589, 2013

6. Harvey KF, Zhang X and Thomas DM: The Hippo pathway and human cancer. Nat Rev Cancer 13: 246-257,2013.

7. Johnson R and Halder G: The two faces of Hippo: Targeting the Hippo pathway for regenerative medicine and cancer treatment. Nat Rev Drug Discov 13: 63-79, 2014.

8. Bertini E, Oka T, Sudol M, Strano S and Blandino G: YAP: At the crossroad between transformation and tumor suppression. Cell Cycle 8: 49-57, 2009

9. Overholtzer M, Zhang J, Smolen GA, Muir B, Li W, Sgroi DC, Deng CX, Brugge JS and Haber DA: Transforming properties of YAP, a candidate oncogene on the chromosome 11q22 amplicon. Proc Natl Acad Sci USA 103: 12405-12410, 2006.

10. Zender L, Spector MS, Xue W, Flemming P, Cordon-Cardo C, Silke J,Fan ST, Luk JM, Wigler M, Hannon GJ, et al: Identification and validation of oncogenes in liver cancer using an integrative oncogenomic approach. Cell 125: 1253-1267, 2006.

11. Steinhardt AA, Gayyed MF, Klein AP, Dong J, Maitra A, Pan D, Montgomery EA and Anders RA: Expression of Yes-associated protein in common solid tumors. Hum Pathol 39: 1582-1589, 2008.

12. Fernandez-L A, Northcott PA, Dalton J, Fraga C, Ellison D, Angers S, Taylor MD and Kenney AM: YAP1 is amplified and up-regulated in hedgehog-associated medulloblastomas and mediates Sonic hedgehogdriven neural precursor proliferation. Genes Dev 23: 2729-2741, 2009.

13. Xu MZ, Yao TJ, Lee NP, Ng IO, Chan YT, Zender L, Lowe SW, Poon RT and Luk JM: Yes-associated protein is an independent prognostic marker in hepatocellular carcinoma. Cancer 115: 4576-4585, 2009.

14. Zhao B, Wei X, Li W, Udan RS, Yang Q, Kim J, Xie J, Ikenoue T, $\mathrm{Yu} \mathrm{J}, \mathrm{Li} \mathrm{L}$, et al: Inactivation of YAP oncoprotein by the Hippo pathway is involved in cell contact inhibition and tissue growth control. Genes Dev 21: 2747-2761, 2007.

15. Zhang X, George J, Deb S, Degoutin JL, Takano EA, Fox SB; AOCS Study group, Bowtell DD and Harvey KF: The Hippo pathway transcriptional co-activator, YAP, is an ovarian cancer oncogene. Oncogene 30: 2810-2822, 2011.

16. Zhang J, Xu ZP, Yang YC, Zhu JS, Zhou Z and Chen WX: Expression of Yes-associated protein in gastric adenocarcinoma and inhibitory effects of its konckdown on gastric cancer cell proliferation and metastasis. Int J Immunopathol Pharmacol 25: 583-590, 2012
17. Zhang W, Nandakumar N, Shi Y, Manzano M, Smith A, Graham G, Gupta S, Vietsch EE, Laughlin SZ, Wadhwa M, et al: Downstream of mutant KRAS, the transcription regulator YAP is essential for neoplastic progression to pancreatic ductal adenocarcinoma. Sci Sig 7: ra42, 2014

18. Zhang L, Yang S, Chen X, Stauffer S, Yu F, Lele SM, Fu K, Datta K, Palermo N, Chen Y and Dong J: The Hippo pathway effector YAP regulates motility, invasion, and castration-resistant growth of prostate cancer cells. Mol Cell Biol 35: 1350-1362, 2015 .

19. Sheng X, Li WB, Wang DL, Chen KH, Cao JJ, Luo Z, He J, Li MC, Liu WJ and Yu C: YAP is closely correlated with castration-resistant prostate cancer, and downregulation of YAP reduces proliferation and induces apoptosis of PC-3 cells. Mol Med Rep 12: 4867-4876, 2015.

20. Livak KJ and Schmittgen TD: Analysis of relative gene expression data using real-time quantitative PCR and the 2(-Delta Delta C(T)) method. Methods 25: 402-408, 2001.

21. Planque $\mathrm{N}$ and Perbal B: A structural approach to the role of CCN (CYR61/CTGF/NOV) proteins in tumourigenesis. Cancer Cell Int 3: 15, 2003.

22. Lamar JM, Stern P, Liu H, Schindler JW, Jiang ZG and Hynes RO: The Hippo pathway target, YAP, promotes metastasis through its TEAD-interaction domain. Proc Natl Acad Sci USA 109: E2441-E2450, 2012.

23. Dong J, Feldmann G, Huang J, Wu S, Zhang N, Comerford SA, Gayyed MF, Anders RA, Maitra A and Pan D: Elucidation of a universal size-control mechanism in Drosophila and mammals. Cell 130: 1120-1133, 2007.

24. Zhao B, Li L, Wang L, Wang CY, Yu J and Guan KL: Cell detachment activates the Hippo pathway via cytoskeleton reorganization to induce anoikis. Genes Dev 26: 54-68, 2012.

25. Yu FX, Zhao B, Panupinthu N, Jewell JL, Lian I, Wang LH, Zhao J, Yuan H, Tumaneng K, Li H, et al: Regulation of the Hippo-YAP pathway by G-Protein-Coupled receptor signaling. Cell 150: 780-791, 2012

26. Li W, Wang L, Katoh H, Liu R, Zheng P and Liu Y: Identification of a tumor suppressor relay between the FOXP3 and the Hippo pathways in breast and prostate cancers. Cancer Res 71: 2162-2171, 2011.

27. Cordenonsi M, Zanconato F, Azzolin L, Forcato M, Rosato A, Frasson C, Inui M, Montagner M, Parenti AR, Poletti A, et al: The Hippo transducer TAZ confers cancer stem cell-related traits on breast cancer cells. Cell 147: 759-772, 2011.

28. Xia Y, Chang T, Wang Y, Liu Y, Li W, Li M and Fan HY: Correction: YAP promotes ovarian cancer cell tumorigenesis and is indicative of a poor prognosis for ovarian cancer patients. PLoS One 11: e0152712, 2016.

29. Ehmer U and Sage J: Control of proliferation and cancer growth by the Hippo signaling pathway. Mol Cancer Res 14: 127-140, 2016.

30. Zhou Z, Zhu JS, Gao CP, Li LP, Zhou C, Wang H and Liu XG: siRNA targeting YAP gene inhibits gastric carcinoma growth and tumor metastasis in SCID mice. Oncol Lett 11: 2806-2814, 2016.

31. Cheng TY, Wu MS, Hua KT, Kuo ML and Lin MT: Cyr61/CTGF/Nov family proteins in gastric carcinogenesis. World J Gastroenterol 20: 1694-1700, 2014. 